\title{
Bayesian inference of non-linear multiscale model parameters accelerated by a Deep Neural Network
}

\author{
Ling $\mathrm{Wu}^{\mathrm{a}, *}$, Kepa Zulueta Uriondo ${ }^{\mathrm{b}}$, Zoltan Major ${ }^{\mathrm{c}}$, Aitor Arriaga ${ }^{\mathrm{b}}$, Ludovic Noels ${ }^{\mathrm{a}}$ \\ ${ }^{a}$ University of Liege, CM3, Allée de la découverte 9, B-4000 Liège, Belgium \\ ${ }^{b}$ Leartiker Polymer RED, Xemein Etorbidea 12A, 48270 Markina-Xemein, Bizkaia, Spain \\ ${ }^{c}$ Johannes Kepler University Linz, Institute of Polymer Product Engineering, Altenbergerstrasse 69, 4040 \\ Linz, Austria
}

\begin{abstract}
We develop a Bayesian Inference (BI) of a non-linear multiscale model and material parameters using experimental composite coupons tests as observation data. In particular we consider non-aligned Short Fibers Reinforced Polymer (SFRP) as a composite material system and Mean-Field Homogenization (MFH) as a multiscale model. Although MFH is computationally efficient, when considering non-aligned inclusions, the evaluation cost of a non-linear response for a given set of model and material parameters remains too prohibitive to be coupled with the sampling process required by the BI. Therefore, a Neural-Networktype (NNW) is first trained using the MFH model, and is then used as a surrogate model during the BI process, making the identification process affordable.
\end{abstract}

Keywords: Multiscale, Composites, Bayesian inference, Neural Network, Non-linear

\section{Introduction}

Short Fibers Reinforced Polymer (SFRP) composites are nowadays commonly used in several industrial applications, increasing the need for computationally efficient modeling tools. Because of the heterogeneous nature of the material, multiscale methods are favored [1], in particular in the non-linear range, in order to capture the effects of the micro-structure

\footnotetext{
${ }^{*}$ Corresponding author: L.Wu@ulg.ac.be

Email address: Zoltan.Major@jku.at (Zoltan Major)
} 
geometrical parameters, such as the inclusions aspect ratio, orientation and spatial distributions, and of the micro-constituents non-linear material responses.

Mean-Field Homogenization (MFH) is an efficient semi-analytical multiscale method which extends the Eshelby single inclusion solution [2] to multiple-inclusion interactions, such as in the Mori-Tanaka (M-T) scheme [3]. In the non-linear range, MFH revolves around the definition of a Linear Comparison Composite (LCC) [4-6] as a virtual heterogeneous linear material system, allowing to extend linear theories while keeping a good to excellent accuracy. Two-step MFH has also been developed in the context of non-aligned inclusions using an orientation distribution function (ODF) to describe their misalignment. Homogenization is thus performed in two stages, first on pseudo-grains of aligned inclusions, and then by weighting the pseudo-grain responses with the ODF $[7,8]$. Since the manufacturing process induces a variation of the ODF along the sample thickness, this 2-step homogenization has to be repeated on different layers to account for the so-called skin-core effect.

One of the difficulty with multiscale methods in general, and MFH in particular, is to identify the parameters defining the microstructure such as fibers aspect ratio distribution, volume fraction, ODF, but also the material laws parameters modeling the phases non-linear responses. On the one hand, fibers ODF, or again volume fraction can be experimentally measured [9] or predicted by a process numerical simulation [9]. However, although the fiber aspect ratio distribution can be experimentally measured, when using the ODF in the context of a two-step MFH, it is convenient to consider a unique "effective" aspect ratio, which has yet to be identified. On the other hand, although fibers properties of SFRP are usually known, the matrix material properties is sensitive to the process conditions and cannot always be directly identified because of the difficulty to produce samples whose material response is exactly the same as the polymeric matrix of the composite material; the parameters of the polymeric material models are thus generally obtained through an inverse identification process from composite coupons tests.

Therefore some micro-structure geometrical parameters, such as the effective aspect ratio, and some phases material parameters, such as the matrix model parameters, should be 
inferred from composite experimental responses. However, because of the number of parameters arising in the non-linear range, this identification requires several loading conditions to be performed, and a unique set of parameters cannot reproduce all the experimental tests because of the model limitations. Besides, the composite responses are inevitably entailed by experimental errors. These difficulties can be circumvented by considering a Bayesian Inference (BI) [10] for which uncertainties in the inferred model parameters arise from the identification process itself under the form of a so-called posterior Probability Density Function $(\mathrm{PDF})$. This posterior PDF of the parameters set is the correction of the initial belief one has on the parameters set distribution, or prior distribution, by a likelihood function evaluated using different observation data, i.e. here the experimental results.

BI was extensively applied to identify the parameters of either, possibly non-linear, homogeneous material models $[11-13$, e.g. $]$ or, generally linear, homogenized composite material models $[14,15$, e.g]. The inference of multi-scale model parameters is more difficult because of its inherent computational cost making the evaluation of the likelihood time consuming when considering a random sampling process. Although in [16] the authors have inferred the two-step MFH parameters of SFRP, this was limited to the linear range because of the computational cost bottleneck: when considering a two-step homogenization as a non-linear multiscale model, one simple analysis needs around one minute of computation but hundreds of thousands of simulations are required for the sampling process. In order to make BI computationally affordable in the non-linear range, in this work, the two-step MFH model is substituted by a surrogate model during the random sampling process. The surrogate model can be any kind of feasible high dimensional nonlinear mapping. Depending on the features of the input and output, typical surrogate models can be constructed by linear combinations of well chosen high dimensional nonlinear base functions. However, a certain experience is required to choose the proper dimension of the function base and the order of the nonlinear functions. Neural Networks (NNW) can theoretical carry out any high dimensional nonlinear mapping if the NNW is designed with enough hidden layers and enough neurons on them. With the help of open NNW library, the experience required to build a high dimensional nonlinear surrogate model is minimized, which offers a more user-friendly and general 
solution. Artificial Neural Networks (ANNW) have been used in the literature to reproduce the homogenized behavior predicted by computational homogenization methods, either by approximating the strain energy density surface $[17,18$, e.g.] or the stress-strain responses $[19,20$, e.g.]. The latter approach is chosen in the paper, and a NNW is trained by the twostep MFH for different sets of micro-structure geometrical and material parameters, and for different loading directions. The likelihood function is then constructed by considering Gaussian noise [11-13] as an error function [15] evaluated from the experimental observation on $40 \%$ of weight GF reinforced PA06 (PA06-GF40) coupon tests. The BI is then conducted using a Metropolis-Hastings (MCMC) random walk during which the likelihood is evaluated using the NNW as surrogate.

The organization of the paper is as follows. The non-linear two-step MFH model is described in Section 2. Section 3 details the construction of the NNW surrogate model and Section 4 summarizes the experimental tests conducted on PA06-GF40 composite coupons. Finally, the BI is presented in Section 5 and the results analysis in Section 6. Conclusions are drawn in Section 7.

\section{Mean-field homogenization for non-aligned short fiber-reinforced composites}

The finite element analysis of structures made of heterogeneous materials can be performed in a homogenization-based multiscale approach, in which the relation between the macro-strains $\varepsilon_{\mathrm{M}}$ and stresses $\boldsymbol{\sigma}_{\mathrm{M}}$ is transformed into a relation between the averaged values of the local strain tensor $\varepsilon_{\mathrm{m}}$ and of the local stress tensor $\boldsymbol{\sigma}_{\mathrm{m}}$ on a micro-scale volume $\boldsymbol{\omega}$, with

$$
\varepsilon_{\mathrm{M}}=\left\langle\varepsilon_{\mathrm{m}}\right\rangle_{\omega} \quad \text { and } \quad \boldsymbol{\sigma}_{\mathrm{M}}=\left\langle\boldsymbol{\sigma}_{\mathrm{m}}\right\rangle_{\omega}
$$

where $\langle f(\boldsymbol{x})\rangle_{\omega}=\frac{1}{V_{\omega}} \int_{\omega} f(\boldsymbol{x}) \mathrm{d} V$, with $V_{\omega}$ the volume of $\omega$.

With a view to the homogenization of SFRP composites, the general equations for twophase composites with aligned uniform inclusions are first presented, the two-step homogenization method for non-aligned inclusions is then summarized before being extended to account for skin-core effect. Finally the material models used for the different phases are 
summarized.

\subsection{Mean-Field Homogenization (MFH) for two-phase composites}

\subsubsection{Mean-field equations for two-phase linear elastic materials}

Considering a two-phase composite material with the respective volume fractions $v_{0}+v_{\mathrm{I}}=$ 1, where the subscript 0 refers to the matrix and the subscript I to the aligned inclusions, the volume averages over the micro-scale volume $\omega$, Eqs. (1), can be explicitly expressed in terms of the volume averages over the two phases $\omega_{0}$ and $\omega_{\mathrm{I}}$, as

$$
\varepsilon_{\mathrm{M}}=v_{0} \varepsilon_{0}+v_{\mathrm{I}} \varepsilon_{\mathrm{I}} \quad \text { and } \quad \boldsymbol{\sigma}_{\mathrm{M}}=v_{0} \boldsymbol{\sigma}_{0}+v_{\mathrm{I}} \boldsymbol{\sigma}_{\mathrm{I}}
$$

where $\bullet_{i}$ denotes the volume average over the phase $\omega_{i}$, i.e. $\left\langle\bullet_{\mathrm{m}}\right\rangle_{\omega_{i}}$, for conciseness.

In the linear elastic range, the system of Eqs. (2) is completed by assuming a relationship between the average strains of the different phases using a strain concentration tensor $\mathbb{B}^{\epsilon}$, which is defined through the elastic tensors $\mathbb{C}_{i}^{\mathrm{el}}$ in phase $\omega_{i}$, and reads

$$
\varepsilon_{\mathrm{I}}=\mathbb{B}^{\epsilon}\left(\mathrm{I}, \mathbb{C}_{0}^{\mathrm{el}}, \mathbb{C}_{\mathrm{I}}^{\mathrm{el}}\right): \varepsilon_{0},
$$

where "I" represents the geometry of the inclusions. Using linear elastic constitutive laws $\boldsymbol{\sigma}_{i}=\mathbb{C}_{i}^{\mathrm{el}}: \boldsymbol{\varepsilon}_{i}$, the set of Eqs. (2) and (3) can be rewritten in a general constitutive expression for linear elastic composites as

$$
\boldsymbol{\sigma}_{\mathrm{M}}=\mathbb{C}_{\mathrm{M}}^{\mathrm{el}}\left(\mathrm{I}, \mathbb{C}_{0}^{\mathrm{el}}, \mathbb{C}_{\mathrm{I}}^{\mathrm{el}}, v_{\mathrm{I}}\right): \varepsilon_{\mathrm{M}}
$$

with $\mathbb{C}_{\mathrm{M}}^{\mathrm{el}}=\left[v_{\mathrm{I}} \mathbb{C}_{\mathrm{I}}^{\mathrm{el}}: \mathbb{B}^{\epsilon}\left(\mathrm{I}, \mathbb{C}_{0}^{\mathrm{el}}, \mathbb{C}_{\mathrm{I}}^{\mathrm{el}}\right)+v_{0} \mathbb{C}_{0}^{\mathrm{el}}\right]:\left[v_{\mathrm{I}} \mathbb{B}^{\epsilon}\left(\mathrm{I}, \mathbb{C}_{0}^{\mathrm{el}}, \mathbb{C}_{\mathrm{I}}^{\mathrm{el}}\right)+v_{0} \mathbb{I}\right]^{-1}$, where $(\mathbb{I})_{i j k l}$ is the identity fourth-order tensor.

\subsubsection{Mean-field equations for two-phase elasto-plastic materials}

For the composites whose phases experience elasto-plastic deformations, $\mathrm{MFH}$ is carried out in an incremental form through a so-called Linear Comparison Composite (LCC) [21, 22]. The LCC is a virtual linear heterogeneous material whose constituents behaviors are defined by virtual elastic operators matching the linearized behaviors of the real composite material 


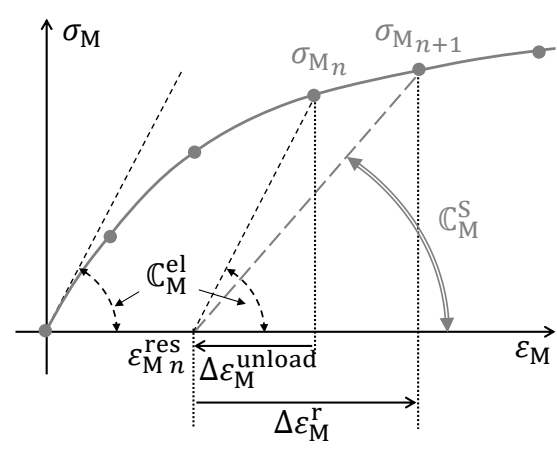

(a) Composite

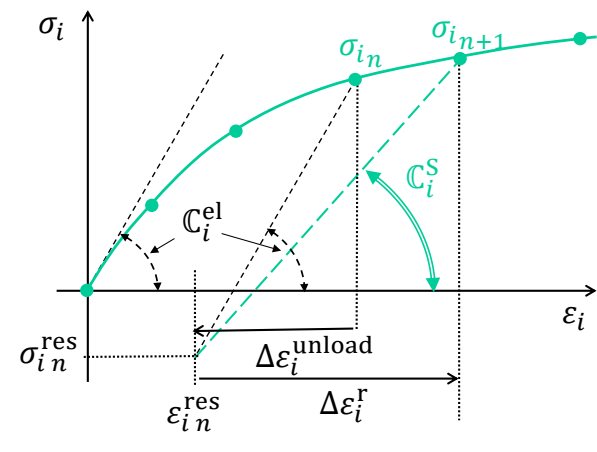

(b) Phase $\omega_{i}$

Figure 1: Definition of the LCC in the incremental-secant method for elasto-plastic composites.

constituents at a given strain state. Therefore, by considering the virtual elastic operators $\mathbb{C}_{0}^{\mathrm{LCC}}$ of the matrix phase and $\mathbb{C}_{\mathrm{I}}^{\mathrm{LCC}}$ of the inclusions phase, the MFH equations of a linear composite material can be applied on the average incremental strains in the two phases; while the homogenized stress relation, Eq. (2a) still holds, the homogenized strain relation, Eq. (2b), and Eq. (3) are rewritten

$$
\begin{aligned}
\Delta \varepsilon_{\mathrm{M}} & =v_{0} \Delta \varepsilon_{0}+v_{\mathrm{I}} \Delta \varepsilon_{\mathrm{I}}, \\
\Delta \varepsilon_{\mathrm{I}} & =\mathbb{B}^{\epsilon}\left(\mathrm{I}, \mathbb{C}_{0}^{\mathrm{LCC}}, \mathbb{C}_{\mathrm{I}}^{\mathrm{LCC}}\right): \Delta \varepsilon_{0} .
\end{aligned}
$$

Among the different linearization techniques developed in order to define the LCC, the incremental-secant approach [6] is considered in this work. During a time increment $\left[t_{n}, t_{n+1}\right]$, the composite material is first subjected to a virtual elastic unloading from the configuration at time $t_{n}$ to reach a residual state so that $\boldsymbol{\sigma}_{\mathrm{M} n}^{\mathrm{res}}=0$, where the subscript "res" refers to the virtually unloaded state. Then, the composite material is loaded to the new configuration at time $t_{n+1}$, see Fig. 1(a).

Since the virtual unloading is elastic, the residual state of the composite is fully determined by the linear elastic MFH formula presented in Section 2.1.1. Then, from the residual state, $\boldsymbol{\varepsilon}_{\mathrm{M} n}^{\mathrm{res}}$ and $\boldsymbol{\sigma}_{\mathrm{M} n}^{\mathrm{res}}=0$, at $t_{n}$, the secant linearization of the non-linear composite material is carried out with the strain increment $\Delta \varepsilon_{\mathrm{M}}^{\mathrm{r}}$ defined such that

$$
\varepsilon_{\mathrm{M}_{n+1}}=\varepsilon_{\mathrm{M}_{n}}^{\mathrm{res}}+\Delta \varepsilon_{\mathrm{M}}^{\mathrm{r}}
$$


where $\varepsilon_{\mathrm{M}_{n+1}}$ is a known value of at the macro-scale. Similarly, the phase strain increments $\Delta \varepsilon_{i}^{\mathrm{r}}$ are defined such that

$$
\varepsilon_{i_{n+1}}=\varepsilon_{i_{n}}^{\mathrm{res}}+\Delta \varepsilon_{i}^{\mathrm{r}}
$$

as illustrated in Fig. 1(b). Finally, in each phase, an incremental-secant operator $\mathbb{C}_{i}^{\mathrm{S}}$ is defined from the phase residual stress-strain states (which do not necessarily vanish) such that

$$
\boldsymbol{\sigma}_{i_{n+1}}=\boldsymbol{\sigma}_{i_{n}}^{\mathrm{res}}+\Delta \boldsymbol{\sigma}_{i}^{\mathrm{r}}, \text { and } \Delta \boldsymbol{\sigma}_{i}^{\mathrm{r}}=\mathbb{C}_{i}^{\mathrm{S}}: \Delta \varepsilon_{i}^{\mathrm{r}}
$$

Therefore, the LCC is defined using the incremental-secant operators $\mathbb{C}_{i}^{S}$, and the set of Eqs. (5-6) is thus rewritten using $\mathbb{C}_{i}^{\mathrm{S}}$ as LCC operator $\mathbb{C}_{i}^{\mathrm{LCC}}$. Finally, the incremental-secant form of Eq. (4) reads

$$
\begin{aligned}
\boldsymbol{\sigma}_{\mathrm{M}_{n+1}} & =\Delta \boldsymbol{\sigma}_{\mathrm{M}}^{\mathrm{r}}=\mathbb{C}_{\mathrm{M}}^{\mathrm{S}}\left(\mathrm{I}, \mathbb{C}_{0}^{\mathrm{S}}, \mathbb{C}_{\mathrm{I}}^{\mathrm{S}}, v_{\mathrm{I}}\right): \Delta \varepsilon_{\mathrm{M}}^{\mathrm{r}}, \text { with } \\
\mathbb{C}_{\mathrm{M}}^{\mathrm{S}} & =\left[v_{\mathrm{I}} \mathbb{C}_{\mathrm{I}}^{\mathrm{S}}: \mathbb{B}^{\epsilon}\left(\mathrm{I}, \mathbb{C}_{0}^{\mathrm{S}}, \mathbb{C}_{\mathrm{I}}^{\mathrm{S}}\right)+v_{0} \mathbb{C}_{0}^{\mathrm{S}}\right]:\left[v_{\mathrm{I}} \mathbb{B}^{\epsilon}\left(\mathrm{I}, \mathbb{C}_{0}^{\mathrm{S}}, \mathbb{C}_{\mathrm{I}}^{\mathrm{S}}\right)+v_{0} \mathrm{I}\right]^{-1}
\end{aligned}
$$

The resolution of the MFH equations (10-11) follows the iterative process detailed in [6]. We note for completness that the residual stress is cancelled in the matrix phase, see details in $[6]$.

\subsection{Strain concentration tensor}

The strain concentration tensors $\mathbb{B}^{\epsilon}\left(\mathrm{I}, \mathbb{C}_{0}^{\mathrm{el}}, \mathbb{C}_{\mathrm{I}}^{\mathrm{el}}\right)$ and $\mathbb{B}^{\epsilon}\left(\mathrm{I}, \mathbb{C}_{0}^{\mathrm{S}}, \mathbb{C}_{\mathrm{I}}^{\mathrm{S}}\right)$ are built upon assumptions. We consider the Mori-Tanaka [3] method (M-T) and Voigt model respectively for two-phase and multi-phase composites. In the following expressions, $\mathbb{C}_{i}$ refers to either $\mathbb{C}_{i}^{\mathrm{el}}$ or $\mathbb{C}_{i}^{\mathrm{S}}$ in the cases of linear elasticity and non-linear elasto-plasticity, respectively.

- The M-T method assumes that the average strain in the matrix phase corresponds to the strain at infinity of the single inclusion solution problem, i.e.

$$
\mathbb{B}^{\epsilon}\left(\mathrm{I}, \mathbb{C}_{0}, \mathbb{C}_{\mathrm{I}}\right)=\left\{\mathbb{I}+\mathbb{S}:\left[\left(\mathbb{C}_{0}\right)^{-1}: \mathbb{C}_{\mathrm{I}}-\mathbb{I}\right]\right\}^{-1}
$$

where the Eshelby tensor $[2] \mathbb{S}\left(\mathrm{I}, \mathbb{C}_{0}^{\mathrm{el}}\right)$ depends on the geometry of the inclusion "I" and on the elastic tensor of the matrix phase $\mathbb{C}_{0}^{\mathrm{el}}$. 
- The Voigt model assumes the same average strain in the different phases, i.e.

$$
\mathbb{B}^{\epsilon}=\mathbb{I} .
$$

\subsection{MFH for multi-phase composite materials}

For short-fiber reinforced composites, the composite material cannot be treated as being two-phase in the MFH process because of the misalignment and of the variation in aspect ratio of the fibers. When considering such a material with inclusions having different orientations or shapes, a two-step homogenization strategy [7, 8] can be adopted.

\subsubsection{Two-step homogenization}
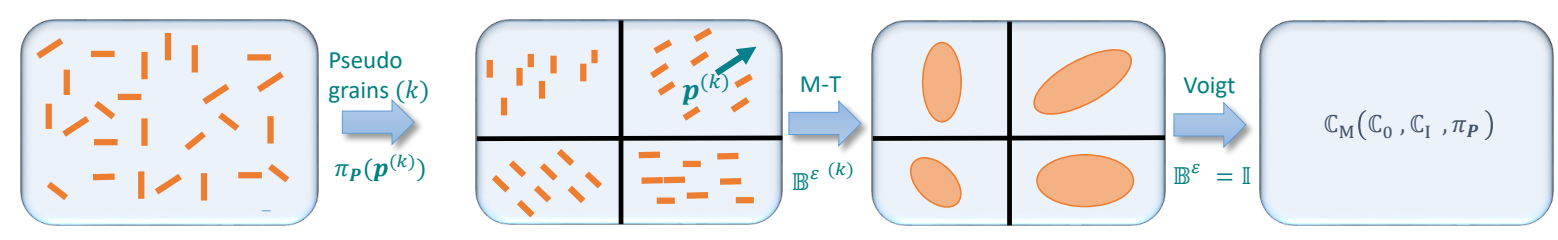

Figure 2: Two-step homogenization: the micro-scale volume element $\omega$ is decomposed into a set of pseudograins $\omega^{(k)}$ homogenized using M-T assumption; the composite behavior is obtained using Voigt assumption.

For a short-fiber reinforced composite material, whose volumes of matrix and short fibers are denoted by $V_{0}$ and $V_{\mathrm{I}}$, respectively, the volume fraction of matrix reads $v_{0}=\frac{V_{0}}{V_{0}+V_{\mathrm{I}}}$. First, it is assumed that all the short fibers are straight and that the aspect ratio of a fiber is defined by its length $l$ over its diameter $d, a_{r}=\frac{l}{d}$. Besides, it is further assumed that all the fibers have the same aspect ratio $A r$, which is an effective value and serves as a material parameter. Finally, the fiber orientation is characterized by a unit vector $\boldsymbol{p}$ oriented along its axis. Therefore, the two-step homogenization process of short fiber reinforced composites can be achieved as illustrated in Fig. 2 and is summarized as follows

- The composite material is treated as an aggregate of pseudo-grains, $\omega^{(k)}(k=1,2, \ldots)$, in which the inclusions "I ${ }^{(k)}$ " have the same aspect ratio $A r$, and the same orientation defined by the direction $\boldsymbol{p}^{(k)}$. All these pseudo-grains see the same volume fraction of matrix $v_{0}$; 
- The homogenization is first performed on each pseudo-grain $\omega^{(k)}$, with the set of Eqs. (5-6) rewritten as

$$
\begin{aligned}
\langle\Delta \boldsymbol{\varepsilon}\rangle_{\omega^{(k)}} & =v_{0}\left\langle\Delta \boldsymbol{\varepsilon}_{0}\right\rangle_{\omega^{(k)}}+v_{\mathrm{I}}\left\langle\Delta \boldsymbol{\varepsilon}_{\mathrm{I}}\right\rangle_{\omega^{(k)}}, \\
\langle\boldsymbol{\sigma}\rangle_{\omega^{(k)}} & =v_{0}\left\langle\boldsymbol{\sigma}_{0}\right\rangle_{\omega^{(k)}}+v_{\mathrm{I}}\left\langle\boldsymbol{\sigma}_{\mathrm{I}}\right\rangle_{\omega^{(k)}}, \\
\left\langle\Delta \boldsymbol{\varepsilon}_{\mathrm{I}}\right\rangle_{\omega^{(k)}} & =\mathbb{B}^{\epsilon}\left(\mathrm{I}^{(k)}, \mathbb{C}_{0}^{\mathrm{S}}, \mathbb{C}_{\mathrm{I}}^{\mathrm{S}}\right):\left\langle\Delta \boldsymbol{\varepsilon}_{0}\right\rangle_{\omega^{(k)}}
\end{aligned}
$$

- The homogenization on the aggregate of pseudo-grains, $\omega^{(k)}(k=1,2, \ldots)$ is then achieved using Voigt strain concentration tensor (13), in which case the set of Eqs. (5-6) is rewritten as

$$
\langle\Delta \varepsilon\rangle_{\omega^{(k)}}=\langle\Delta \varepsilon\rangle_{\omega}=\Delta \varepsilon_{\mathrm{M}} \quad \forall \omega^{(k)}
$$

with the homogenized stress evaluated by

$$
\boldsymbol{\sigma}_{\mathrm{M}}=\langle\boldsymbol{\sigma}\rangle_{\omega}=\sum_{k} v_{\omega^{(k)}}\langle\boldsymbol{\sigma}\rangle_{\omega^{(k)}}
$$

where $v_{\omega^{(k)}}$ is the volume fraction defined by the volume of fibers having an oriented

along a direction $\boldsymbol{p}^{(k)}, V_{\omega^{(k)}}$, over the total volume of fibers $V_{\mathrm{I}}$, e.g. $v_{\omega^{(k)}}=\frac{V_{\omega^{(k)}}}{V_{\mathrm{I}}}$. The values of $v_{\omega^{(k)}}$ can be approximated using a fibers Orientation Distribution Function.

\subsubsection{Orientation Distribution Function (ODF)}

For a collection of fibers, the complete description of their orientations is represented by a probability density function $\pi_{\boldsymbol{P}}(\boldsymbol{p})$, also called Orientation Distribution Function (ODF), such that $\pi_{\boldsymbol{P}}(\boldsymbol{p}) \mathrm{d} \boldsymbol{p}$ is the probability of a fiber to be oriented between $\boldsymbol{p}$ and $\boldsymbol{p}+\mathrm{d} \boldsymbol{p}$ with $\oint \pi_{\boldsymbol{P}}(\boldsymbol{p}) \mathrm{d} \boldsymbol{p}=1$. It is convenient to write the ODF in the spherical coordinates as

$$
\int_{\theta=0}^{\pi} \oint_{\phi=0}^{2 \pi} \pi_{\boldsymbol{P}}(\boldsymbol{p}(\theta, \phi)) \sin (\theta) \mathrm{d} \phi \mathrm{d} \theta=1,
$$

where $\theta$ is the polar angle and $\phi$ is the azimuthal angle. In practice, the $\operatorname{ODF} \pi_{\boldsymbol{P}}(\boldsymbol{p})$ is not always directly available, and it is commonly constructed through a second-order orientation tensor, which reads $[8,23]$,

$$
\boldsymbol{a}=\oint \boldsymbol{p} \otimes \boldsymbol{p} \pi_{\boldsymbol{P}}(\boldsymbol{p}) \mathrm{d} \boldsymbol{p}
$$


More details on the construction of $\pi_{\boldsymbol{P}}(\boldsymbol{p}(\theta, \phi))$ from $\boldsymbol{a}$ can be found in $[8,16]$. Since a constant fiber aspect ratio $A r$ is assumed, the value of $v_{\omega^{(k)}}$, in Eq. (18), can be approximated by the volume fraction of fibers whose orientations are within $\left[\boldsymbol{p}^{(k)}-\frac{1}{2} \Delta \boldsymbol{p}, \boldsymbol{p}^{(k)}+\frac{1}{2} \Delta \boldsymbol{p}\right]$. Using the expression of ODF in the spherical coordinates, Eq. (19), we have

$$
v_{\omega^{(k)}} \approx \int_{\theta^{(k)}-\frac{\Delta \theta}{2}}^{\theta^{(k)}+\frac{\Delta \theta}{2}} \oint_{\phi^{(k)}-\frac{\Delta \phi}{2}}^{\phi^{(k)}+\frac{\Delta \phi}{2}} \pi_{\boldsymbol{P}}(\boldsymbol{p}(\theta, \phi)) \sin (\theta) \mathrm{d} \phi \mathrm{d} \theta,
$$

where $\theta^{(k)}$ and $\phi^{(k)}$ are respectively the polar and azimuthal angles of orientation $\boldsymbol{p}^{(k)}$. The angle increments $\Delta \theta=\pi / N_{\frac{\pi}{2}}$, and $\Delta \phi=\pi / N_{\theta}$ with $N_{\theta}=N_{\frac{\pi}{2}} \sin (\theta)$, are chosen such that the surface of the unit sphere is subdivided into facets of almost equal areas [8].

\subsubsection{Skin-Core effect}

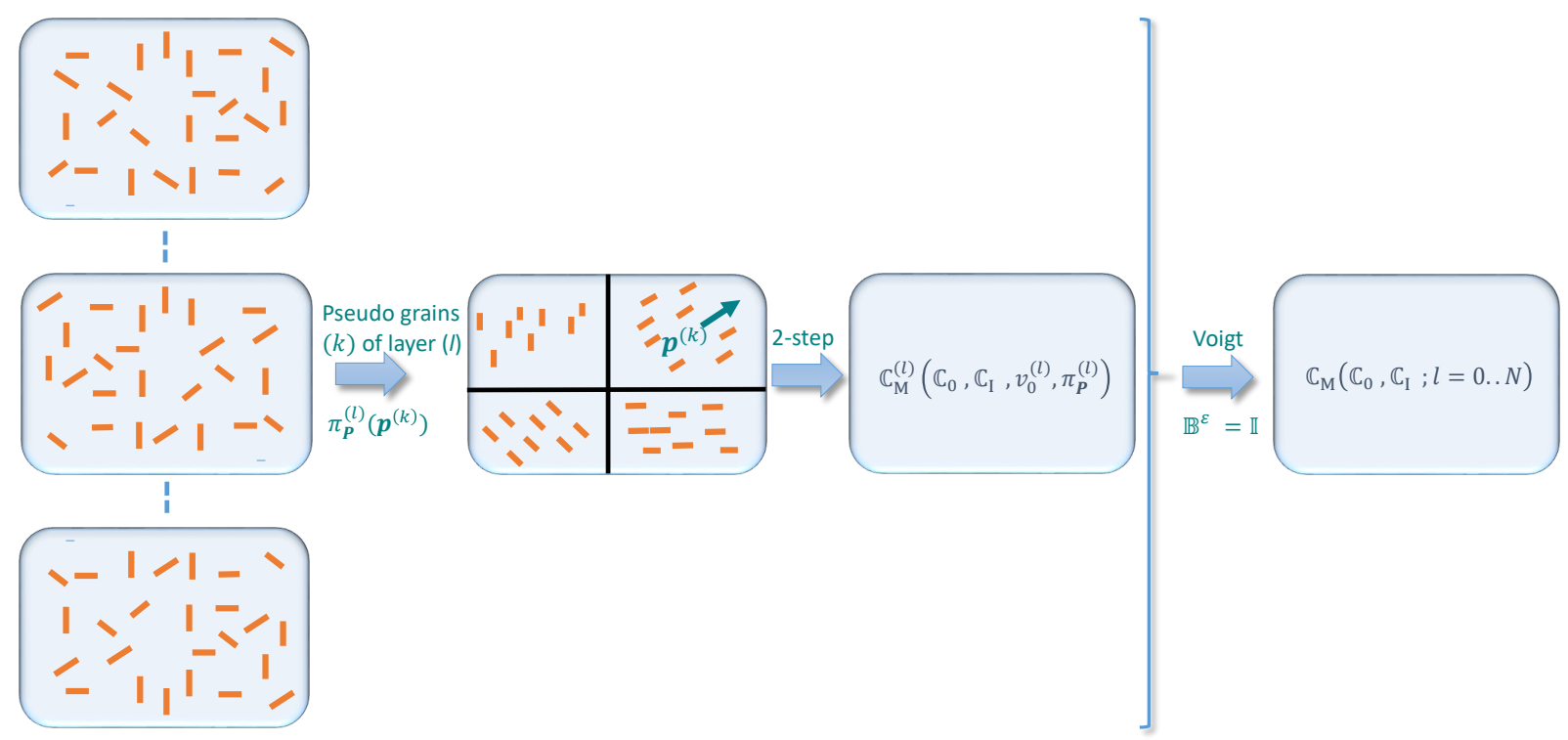

Figure 3: Two-step homogenization with skin-core effect: the micro-scale volume element $\omega$ is decomposed into layers $(l)$; each layer is decomposed into pseudo-grains $\omega^{(k, l)}$ and its homogenized behavior is obtained using the 2-step homogenization; the composite behavior is eventually obtained using Voigt assumption on the different layers.

During the injection molding process, there exists a skin-core effect, in which case the fiber orientation distribution is not uniform across the plate thickness. In this work, the 
plate thickness is divided into $N_{l}$ layers, and the 2-step homogenization is applied in each layer $(l)$ characterized by a distribution $\pi_{\boldsymbol{P}}^{(l)}(\boldsymbol{p})$ and an inclusions volume fraction $v_{\mathrm{I}}^{(l)}$. This 2step homogenization is followed by a Voigt homogenization of the different layers responses, see Fig. 3. This second Voigt assumption is justified because of the similar out-of-plane behavior of the different layers due to the fact that the fibers are mostly oriented in the plane.

With $\omega^{(k ; l)}$ the pseudo-grain $(k)$ of the layer $(l)$, the set of Eqs. (17-18) finally becomes,

$$
\begin{gathered}
\langle\Delta \varepsilon\rangle_{\omega(k ; l)}=\langle\Delta \varepsilon\rangle_{\omega}=\Delta \varepsilon_{\mathrm{M}} \quad \forall \omega^{(k ; l)}, \\
\boldsymbol{\sigma}_{\mathrm{M}}=\langle\boldsymbol{\sigma}\rangle_{\omega}=\sum_{l}^{N_{l}} \sum_{k} v_{\omega(k ; l)}\langle\boldsymbol{\sigma}\rangle_{\omega(k ; l),}
\end{gathered}
$$

where $v_{\omega(k, l)}$ is obtained from Eq. (21) as

$$
v_{\omega^{(k ; l)}} \approx \frac{1}{N_{l}} \int_{\theta^{(k)}-\frac{\Delta \theta}{2}}^{\theta^{(k)}+\frac{\Delta \theta}{2}} \oint_{\phi^{(k)}-\frac{\Delta \phi}{2}}^{\phi^{(k)}+\frac{\Delta \phi}{2}} \pi_{\boldsymbol{P}}^{(l)}(\boldsymbol{p}(\theta, \phi)) \sin (\theta) \mathrm{d} \phi \mathrm{d} \theta .
$$

Remarks: In the considered short-fiber reinforced material, the fiber aspect ratio is not a uniform value. It has however been shown in [16] that the distribution of aspect ratio along the injection direction $\left(\theta=\frac{\pi}{2}, \phi=0\right)$ is representative of the in-plane directions, while the content of fibers along out-of-plane direction is neglectable. Therefore, the approximation in Eq. (21) still holds providing that an effective aspect ratio is used in the model.

\subsection{Phases materials}

The material system is a short E-glass fibers reinforced PA06 polyamide.

- The short glass fibers are modeled using a linear elastic constitutive law with Young's modulus $E_{\mathrm{I}}=72.0 \mathrm{GPa}$ and Poisson coefficient $\nu_{\mathrm{I}}=0.22$.

- The PA06 matrix material is modeled as an elasto-plastic material with Poisson coefficient $\nu_{0}=0.4$. Its Young's modulus $E_{0}$ and initial yielding stress $\sigma_{\mathrm{Y} 0}$ are unknown at this stage and should be inferred. The matrix plastic flow is assumed to follow an isotropic hardening law in terms of the accumulated plastic strain $p$, which reads

$$
R(p)=h p^{m_{1}}\left(1-\exp \left(-m_{2} p\right)\right)
$$


where $h, m_{1}$ and $m_{2}$ are unknown hardening parameters to be inferred.

The unknown material parameters $E_{0}, \sigma_{\mathrm{Y} 0}, h, m_{1}, m_{2}$ and the effect of the effective fiber aspect ratio $A r$ will be identified by Bayesian Inference (BI) using experimental tests conducted on composite coupons. Although the presented two-step homogenization is rather efficient compared to computational homogenization, running this homogenization process during a BI process with a Metropolis-Hastings (MCMC) random walk is only affordable in the linear elastic case. Therefore, in order to carry out the BI in the non-linear range, a Deep Neural Network is adopted as a surrogate model of the two-step homogenization.

\section{Deep Neural Network}

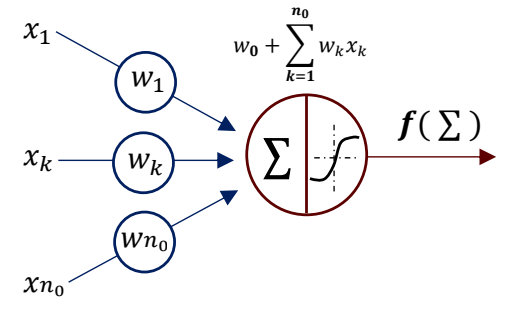

(a)

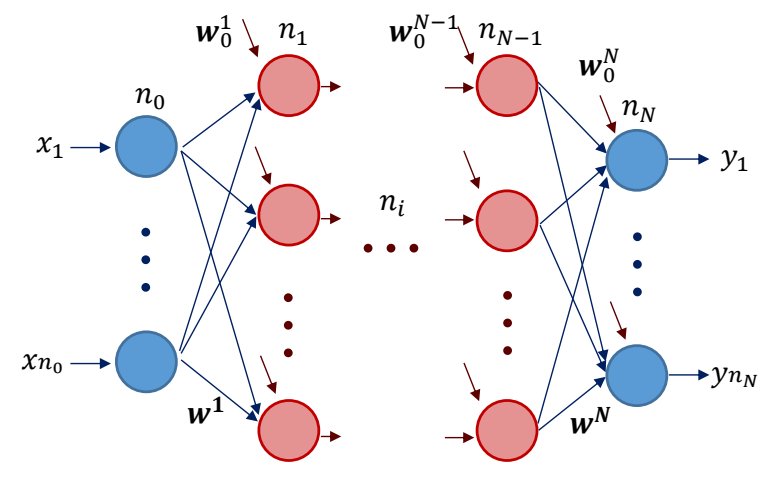

(b)

Figure 4: (a) An artificial neuron; (b) An artificial Neural Network.

Artificial Neural Network (ANNW), or Neural Network (NNW) in short, is a network constructed by artificial neurons, see Fig. 4(a). Artificial neurons first perform a weighted sum operation on input $\left(w_{0}+\sum_{k=1}^{n_{0}} w_{k} x_{k}\right)$, and then produce output through an activation function of the weighted sum $f\left(\sum\right)$. NNW models can be viewed as mathematical models, with a set of algorithms, defining a function $F: \boldsymbol{X} \rightarrow \boldsymbol{Y}$, see Fig. 4(b), in which $\boldsymbol{X}=$ $\left[x_{1}, x_{2}, \ldots, x_{n_{0}}\right]$ and $\boldsymbol{Y}=\left[y_{1}, y_{2}, \ldots, y_{n_{N}}\right]$. 'Deep neural network' is the name used for 'stacked neural network', that is, networks composed of more than one hidden layer, such as in Fig. 4(b) which presents a NNW with $N-1$ hidden layers. 
The training of the NNW refers to regression as supervised learning. Based on a given set of input-output pairs, supervised learning refers to training the weight parameters $w_{k j}^{i}$, with $i=1, \ldots, N ; k=1, \ldots, n_{i-1}$ and $j=1, \ldots, n_{i}$, and bias $w_{0_{j}}^{i}$, with $i=1, \ldots, N$ and $j=$ $1, \ldots, n_{i}$, see the notations in Fig. 4(b), and making the NNW being able to map input to corresponding output. A loss function is defined to measure the difference between the predicted output $\hat{\boldsymbol{Y}}$ and given output $\boldsymbol{Y}$, such as $f_{\text {loss }}=\frac{1}{2}\|\hat{\boldsymbol{Y}}-\boldsymbol{Y}\|^{2}$, where $\|\bullet\|$ refers to the Frobenius norm. Deep learning can be carried out by back-propagation, which updates the values of $\mathbf{w}_{k j}^{i}$ iteratively to minimize the loss function $f_{\text {loss }}$. To this end, the Python library 'Scikit-learn' for NNW regression [24] is used in this work. The architecture of the NNW is obtained by following a trying process: i) starting from a simple architecture; ii) increasing the depth of the network (the number of hidden layers) progressively; and iii) monitoring the improvement of the NNW. The hyperbolic tangent function 'tanh' is chosen as the activation function.

In this work we consider uniaxial tension performed on composite coupons and then use the homogenized strain history (along the loading direction) and material parameters as input, $\boldsymbol{X}=\left[\varepsilon_{\mathrm{M}}, \boldsymbol{\vartheta}\right]$, and as signal output $Y=\left[\sigma_{\mathrm{M}}\right]$, the unidirectional homogenized tensile stress at a given strain $\varepsilon_{\mathrm{M}}$. The material parameters $\boldsymbol{\vartheta}$ correspond to the unknown properties of the elasto-plastic matrix, $E_{0}, \sigma_{\mathrm{Y} 0}, h, m_{1}, m_{2}$, see Eq. (25), completed by the effective short fiber aspect ratio $A r$. Therefore, we have $n_{0}=7$ input for $n_{N}=1$ output, see Fig. 4(b). Finally, a NNW of five hidden layers, with 17 neurons in each hidden layers, is adopted.

Table 1: The ranges of strain and unknown material properties.

\begin{tabular}{c|ccccccc}
\hline $\boldsymbol{\vartheta}$ & $\varepsilon_{\mathrm{M}}[-]$ & $E_{0}[\mathrm{GPa}]$ & $\sigma_{\mathrm{Y} 0}[\mathrm{MPa}]$ & $h[\mathrm{MPa}]$ & $m_{1}[-]$ & $m_{2}[-]$ & $A r[-]$ \\
\hline Ranges & {$[0 \sim 0.04]$} & {$[2 \sim 7]$} & {$[0.01 \sim 70]$} & {$[0.01 \sim 120]$} & {$[0.0001 \sim 1]$} & {$[1 \sim 800]$} & {$[5 \sim 25]$} \\
\hline
\end{tabular}

In order to substitute the multiscale model by a NNW surrogate, the training and testing data of the NNW are achieved using unidirectional tensile simulations obtained with the two-step MFH method presented in Section 2.3 for different realizations of the material parameters $\boldsymbol{\vartheta}$. For each simulation, the strain $\varepsilon_{\mathrm{M}}$ increases from zero to a given value and the material parameters are taken randomly from the given ranges listed in Table 1 . The bounds 
of $E_{0}$ and $A_{r}$ are chosen with respect to the identification process conducted [16] for the same material system, but limited to the linear range, in which the inferred values are within these ranges. The bounds of the other parameters correspond to extreme values by lack of prior knowledge. During each simulation, 70 strain-stress points are recorded: together with the material parameters, they correspond to 70 input and output data pairs of the NNW. Only a few hundreds of simulations (typically $<500$ ) were needed for the designed NNW to be trained (i.e. the mean squared error is lower than $0.5 \times 10^{-4}$ ). Compared to the hundreds of thousands of simulations required for a MCMC random walk, the total computation time is reduced drastically.

\section{Experimental tests}

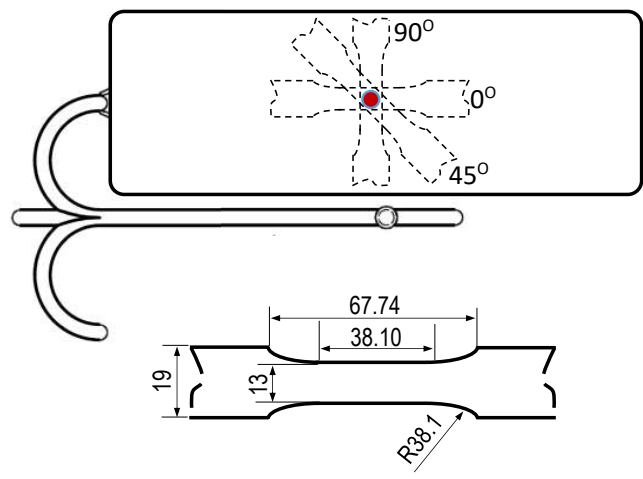

(a) Coupons

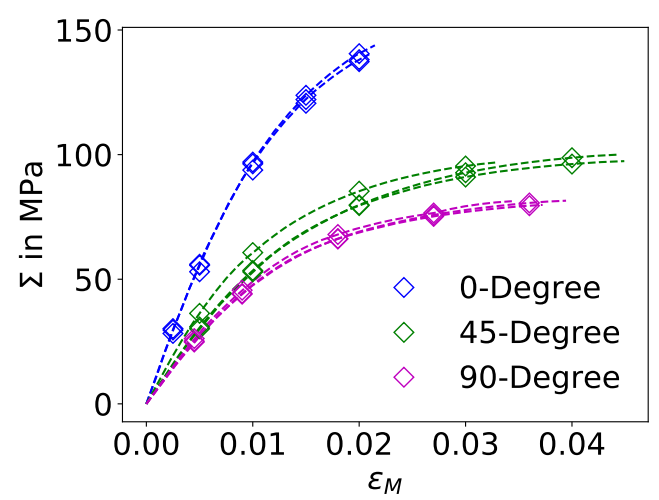

(b) Uniaxial strain-stress curves

Figure 5: (a) Locations and geometries (in $\mathrm{mm}$ ) of the tested coupons along $0^{\circ}, 45^{\circ}$ and $90^{\circ}$ Directions; (b) Unidirectional tensile results of $0^{\circ}, 45^{\circ}$ and $90^{\circ}$.

The $40 \%$ of weight fraction short E-glass fibers (GF) reinforced PA06 composite (PA06GF40) plates of $304.8 \times 101.6 \times 3.65 \pm 0.02 \mathrm{~mm}^{3}$ were manufactured by injection molding with an Engel Insert v200 Single machine. The nozzle temperature has been set at $270^{\circ} \mathrm{C}$ and the mold's temperature at $90^{\circ} \mathrm{C}$. The plates have been injected in 2 seconds under a pressure of 110 bar and the 50-bar packing pressure has been applied during 50 seconds. Coupons were then cut from the plates along 3 different Directions, $0^{\circ}, 45^{\circ}$ and $90^{\circ}$, and 
Table 2: Orientation tensor $\boldsymbol{a}$ and fiber volume fraction $v_{\mathrm{I}}$ at center location for the PA06-GF40 plate: global values $\boldsymbol{a}^{\text {thickness }}$ and $v_{\mathrm{I}}^{\text {thickness }}$ when considering the full thickness and the layer values $\boldsymbol{a}^{(l)}$ and $v_{\mathrm{I}}^{(l)}$ when considering 11 layers to account for the skin-core effect.

\begin{tabular}{|c|c|c|c|c|c|c|c|}
\hline$a=$ & {$\left[a_{11}\right.$} & $a_{22}$ & $a_{33}$ & $a_{12}$ & $a_{23}$ & $\left.a_{13}\right]$ & $v_{\mathrm{I}}$ \\
\hline $\boldsymbol{a}^{\text {thickness }}=$ & {$[0.747$} & 0.204 & 0.049 & 0.031 & -0.0032 & $-0.005]$ & 0.2214 \\
\hline $\boldsymbol{a}^{(1)}=$ & {$[0.851$} & 0.115 & 0.033 & 0.0045 & 0.001 & $-0.0005]$ & 0.2343 \\
\hline $\boldsymbol{a}^{(2)}=$ & {$[0.867$} & 0.096 & 0.037 & 0.0181 & 0.0006 & $-0.005]$ & 0.2323 \\
\hline $\boldsymbol{a}^{(3)}=$ & {$[0.844$} & 0.109 & 0.047 & 0.0261 & 0.0005 & $-0.008]$ & 0.2271 \\
\hline $\boldsymbol{a}^{(4)}=$ & {$[0.817$} & 0.134 & 0.049 & 0.0211 & 0.0009 & $-0.012]$ & 0.2227 \\
\hline $\boldsymbol{a}^{(5)}=$ & {$[0.680$} & 0.260 & 0.060 & 0.0360 & 0.0017 & $-0.036]$ & 0.2035 \\
\hline $\boldsymbol{a}^{(6)}=$ & {$[0.227$} & 0.674 & 0.099 & 0.125 & -0.0134 & $-0.0092]$ & 0.1950 \\
\hline $\boldsymbol{a}^{(7)}=$ & {$[0.648$} & 0.294 & 0.059 & 0.0358 & -0.0098 & $0.0237]$ & 0.2032 \\
\hline $\boldsymbol{a}^{(8)}=$ & {$[0.818$} & 0.139 & 0.044 & 0.0063 & -0.0040 & $0.0026]$ & 0.2240 \\
\hline $\boldsymbol{a}^{(9)}=$ & {$[0.857$} & 0.1054 & 0.038 & 0.0195 & -0.0027 & $-0.0003]$ & 0.2308 \\
\hline $\boldsymbol{a}^{(10)}=$ & {$[0.878$} & 0.092 & 0.031 & 0.0126 & -0.0016 & $-0.0022]$ & 0.2364 \\
\hline $\boldsymbol{a}^{(11)}=$ & {$[0.835$} & 0.134 & 0.030 & 0.0145 & -0.0068 & $-0.0036]$ & 0.2261 \\
\hline
\end{tabular}

centered on the plate as illustrated, with their main dimensions adapted from the ASTM D638, in Fig. 5(a).

Quasi-static uniaxial tensile tests have been performed in a MTS Insight electromechanical actuator at $23^{\circ} \mathrm{C}$ and at a strain rate of $1 \mathrm{~mm} \cdot \mathrm{min}^{-1}$. The strain measurements were carried out by means of a MTS Clip On extensometer with the error of resolution being below $0.009 \%$. The resulting uniaxial tensile strain-stress curves are reported in Fig. 5(b).

The fibers orientations have been characterized by Computed Tomography (CT) technique on volume samples of $2 \times 2 \times 3.2 \mathrm{~mm}^{3}$ at the plate center while the volume fraction has been estimated from the mass fraction measured using the pyrolysis technique, see details in [16]. The second-order orientation tensors $\boldsymbol{a}^{\text {thickness }}$ and the volume fraction $v_{\mathrm{I}}^{\text {thickness }}$ when considering the full thickness are reported in Table 2. The CT-scan measurements are then used to evaluate the orientation tensor $\boldsymbol{a}^{(l)}$ and volume fraction $v_{\mathrm{I}}^{(l)}$ on 11 layers accross the thickness to account for the skin-core effect. 


\section{Bayesian inference of the multiscale parameters and matrix properties}

Bayesian Inference is a statistical analysis approach based on Bayes' theorem which states that the posterior probability of a random parameters vector $\boldsymbol{\alpha} \in \mathbb{R}^{n}$ for given observations of another random vector $\boldsymbol{\beta} \in \mathbb{R}^{m}, \pi(\boldsymbol{\alpha} \mid \boldsymbol{\beta})$, is proportional to the prior probability $\pi_{\text {prior }}(\boldsymbol{\alpha})$ multiplied by the likelihood of $\boldsymbol{\beta}$ for given observations of $\boldsymbol{\alpha}$, where $\pi(\bullet)(\pi(\bullet \bullet \bullet))$ denotes a (conditional) Probability Density Function (PDF).

The prior distribution $\pi_{\text {prior }}(\boldsymbol{\alpha})$ reflects the initial belief or knowledge one has on $\boldsymbol{\alpha}$. The likelihood function is defined from observations of $\boldsymbol{\beta}$ by a conditional $\operatorname{PDF} \pi(\boldsymbol{\beta} \mid \boldsymbol{\alpha})$, which is constructed from the different observation data. The conditional PDF $\pi_{\text {post }}(\boldsymbol{\alpha} \mid \boldsymbol{\beta})$ is the posterior distribution of the random vector $\boldsymbol{\alpha}$ that accounts for the observation data. Since $\pi(\boldsymbol{\beta})$ is a constant for given observations, we simply write

$$
\pi_{\text {post }}(\boldsymbol{\alpha} \mid \boldsymbol{\beta}) \propto \pi(\boldsymbol{\beta} \mid \boldsymbol{\alpha}) \pi_{\text {prior }}(\boldsymbol{\alpha})
$$

\subsection{Stress noise-based inference}

For uni-axial tensile loading, the predictions of two-step MFH model with skin-core effect $\Phi_{\mathrm{MFH}}\left(\varepsilon_{\mathrm{M}}, \boldsymbol{\vartheta}\right)$ reads

$$
\sigma_{\mathrm{M}}\left(\varepsilon_{\mathrm{M}}, \boldsymbol{\vartheta}\right)=\Phi_{\mathrm{MFH}}\left(\varepsilon_{\mathrm{M}}, \boldsymbol{\vartheta}\right)
$$

where $\vartheta$ represents the material parameters, which include the matrix material properties and the effective short fiber aspect ratio, to be inferred. The relation between a stress measurement $\Sigma$ and the model response is written as

$$
\Sigma=\sigma_{\mathrm{M}}+\omega_{\Sigma}=\Phi_{\mathrm{MFH}}\left(\varepsilon_{\mathrm{M}}, \boldsymbol{\vartheta}\right)+\omega_{\Sigma}
$$

where $\omega_{\Sigma}$ is the stress noise assumed to follow a Gaussian distribution $\mathcal{N}\left(x \mid 0, s_{\Sigma}^{2}\right)$ with

$$
\mathcal{N}\left(x \mid y, s_{\Sigma}^{2}\right)=\frac{1}{s_{\Sigma} \sqrt{2 \pi}} \exp \left[-\frac{1}{2}\left(\frac{x-y}{s_{\Sigma}}\right)^{2}\right] .
$$

Therefore, the conditional distribution of stress reads

$$
\pi\left(\Sigma \mid \varepsilon_{\mathrm{M}}, \boldsymbol{\vartheta}\right)=\mathcal{N}\left(\Sigma-\Phi_{\mathrm{MFH}}\left(\varepsilon_{\mathrm{M}}, \boldsymbol{\vartheta}\right) \mid 0, s_{\Sigma}^{2}\right)
$$




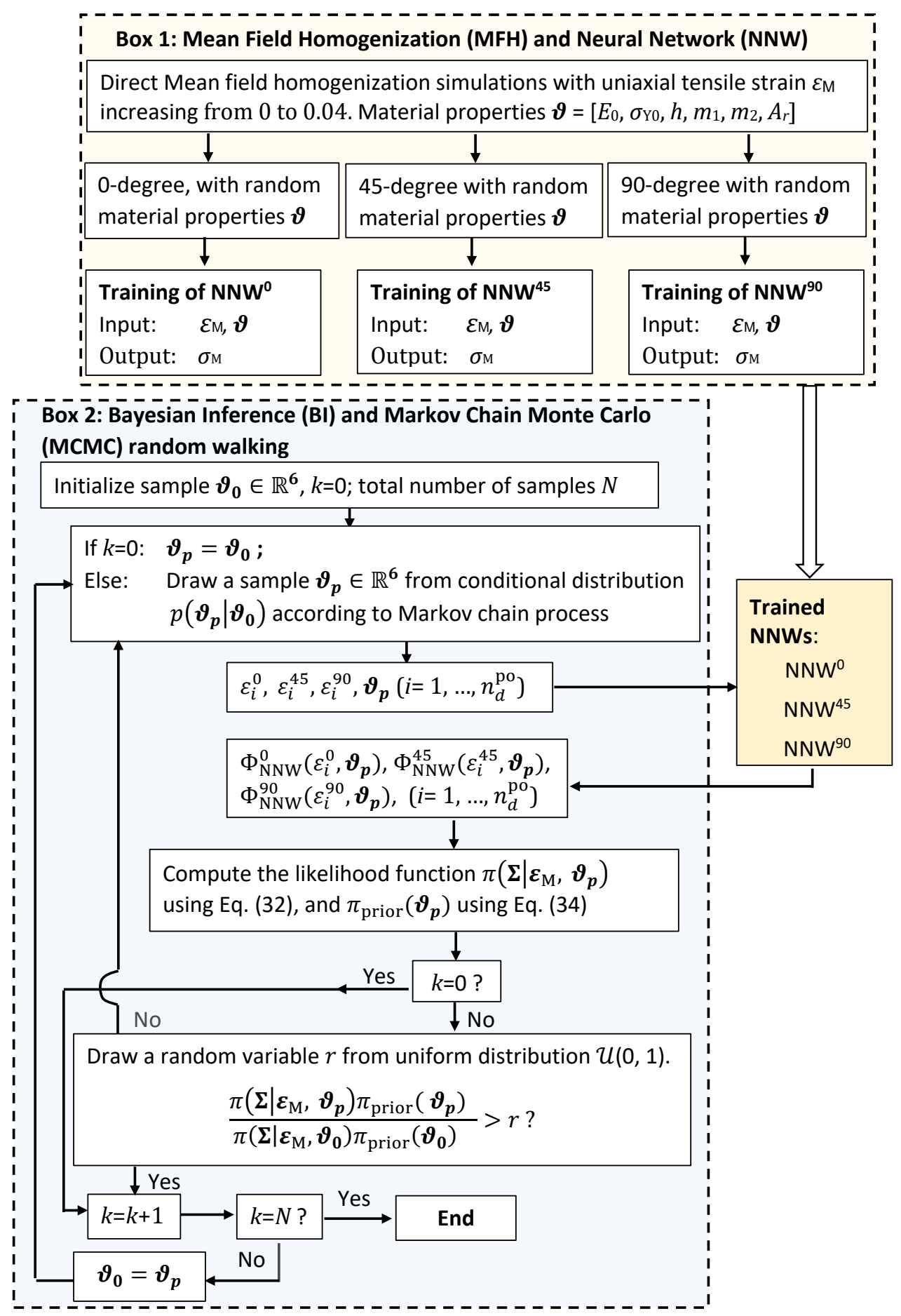

Figure 6: The Baysian inference of the multiscale model $\Phi_{\mathrm{MFH}}$ parameters accelerated by the surrogate model $\Phi_{\text {NNW }}$. 


\subsection{Bayesian inference using the surrogate model}

Since the computational time of the two-step MFH process makes a MCMC sampling unaffordable, in particular when accounting for the skin-core effect, NNW is used as a surrogate $\Phi_{\mathrm{NNW}}$ of $\mathrm{MFH}$ model $\Phi_{\mathrm{MFH}}$ in order to perform the BI, see Fig. 6 .

Table 3: Experimental strain - stress observations.

\begin{tabular}{c|cc|cccc}
\hline \multirow{2}{*}{ Direction } & Strain $\varepsilon_{\mathrm{M}}$ & {$[-]$} & Stress $\boldsymbol{\Sigma}$ & {$[\mathrm{MPa}]$} & & \\
\hline \multirow{4}{*}{$0^{\circ}$} & $\varepsilon_{1}^{0}$ & 0.0025 & $\Sigma_{1}^{0}$ & 28.3 & 29.6 & 30.2 \\
& $\varepsilon_{2}^{0}$ & 0.005 & $\Sigma_{2}^{0}$ & 53.0 & 55.6 & 56.0 \\
& $\varepsilon_{3}^{0}$ & 0.01 & $\Sigma_{3}^{0}$ & 93.8 & 97.0 & 96.3 \\
& $\varepsilon_{4}^{0}$ & 0.015 & $\Sigma_{4}^{0}$ & 120.6 & 123.8 & 122.1 \\
& $\varepsilon_{5}^{0}$ & 0.02 & $\Sigma_{5}^{0}$ & 137.3 & 140.5 & 138.1 \\
\hline \multirow{4}{*}{$45^{\circ}$} & $\varepsilon_{1}^{45}$ & 0.005 & $\Sigma_{1}^{45}$ & 30.1 & 30.9 & 36.3 \\
& $\varepsilon_{2}^{45}$ & 0.01 & $\Sigma_{2}^{45}$ & 53.0 & 53.5 & 60.7 \\
& $\varepsilon_{3}^{45}$ & 0.02 & $\Sigma_{3}^{45}$ & 79.6 & 79.8 & 85.3 \\
& $\varepsilon_{4}^{45}$ & 0.03 & $\Sigma_{4}^{45}$ & 91.0 & 92.7 & 95.4 \\
& $\varepsilon_{5}^{45}$ & 0.04 & $\Sigma_{5}^{45}$ & 96.2 & 98.8 & - \\
\hline \multirow{6}{*}{$90^{\circ}$} & $\varepsilon_{1}^{90}$ & 0.0045 & $\Sigma_{1}^{90}$ & 25.2 & 24.8 & 26.2 \\
& $\varepsilon_{2}^{90}$ & 0.009 & $\Sigma_{2}^{90}$ & 44.3 & 44.0 & 45.6 \\
& $\varepsilon_{3}^{90}$ & 0.018 & $\Sigma_{3}^{90}$ & 66.1 & 66.3 & 68.0 \\
& $\varepsilon_{4}^{90}$ & 0.027 & $\Sigma_{4}^{90}$ & 75.2 & 75.8 & 76.6 \\
& $\varepsilon_{5}^{90}$ & 0.036 & $\Sigma_{5}^{90}$ & 79.5 & 80.6 & - \\
\hline
\end{tabular}

In order to minimize the number of training data for NNW, three NNWs are trained for the three loading Directions, $0^{\circ}, 45^{\circ}, 90^{\circ}$, respectively, as summarized in Box 1 of Fig. 6 . The observations are the unidirectional tensile experimental data points; practically, only the experimental data points marked in Fig. 5(b) are used and are reported in Table 3. Therefore, depending on the loading Direction, Eq. (30) becomes

$$
\pi\left(\Sigma_{i}^{d} \mid \varepsilon_{i}^{d}, \boldsymbol{\vartheta}\right)=\mathcal{N}\left(\Sigma_{i}^{d}-\Phi_{\mathrm{NNW}}^{d}\left(\varepsilon_{i}^{d}, \boldsymbol{\vartheta}\right) \mid 0, s_{\Sigma_{i}^{d}}^{2}\right)
$$


where the superscript $d=0,45,90$ refers to the tensile loading and coupon Direction, and where the subscript $i$ is used to indicate the stress variance $s_{\Sigma_{i}^{d}}$ obtained from the experimental curves at a given strain $\varepsilon_{i}^{d}$, the subscript " $\mathrm{M}$ " being omitted for conciseness.

\subsubsection{Likelihood function}

In order to construct the likelihood, it is assumed that each tensile test is independent from the other ones. This is justified by the fact that the samples are extracted from different composite plates. Using the observations reported in Table 3 and the theory of conditional independence [25], the final likelihood function is obtained from Eq. (31) and reads ${ }^{1}$

$$
\pi\left(\boldsymbol{\Sigma} \mid \varepsilon_{\mathrm{M}}, \boldsymbol{\vartheta}\right)=\prod_{d, i, j} \mathcal{N}\left(\Sigma_{i, j}^{d}-\Phi_{\mathrm{NNW}}^{d}\left(\varepsilon_{i}^{d}, \boldsymbol{\vartheta}\right) \mid 0, s_{\Sigma_{i}^{d}}^{2}\right)
$$

where $\varepsilon_{\mathrm{M}}$ and $\Sigma$ represent the strain, $\varepsilon_{i}^{d}$, and stress, $\Sigma_{i, j}^{d}$, observations listed in Table 3, with $i=1, \ldots, n_{d}^{\mathrm{po}}, n_{d}^{\mathrm{po}}$ being the considered points number on a stress-strain curve, and $j=1, \ldots, n_{d, i}^{\mathrm{ob}}, n_{d, i}^{\mathrm{ob}}$ being the number of stress observations corresponding to the strain $\varepsilon_{i}^{d}$. The standard deviations are obtained from 43 pairs of observations $\left(\varepsilon_{i}^{d}, \Sigma_{i, j}^{d}\right)$ listed in Table 3 as

$$
s_{\Sigma_{i}^{d}}^{2}=\frac{1}{n_{d, i}^{\mathrm{ob}}-1} \sum_{j=1}^{n_{d, i}^{\mathrm{ob}}}\left(\Sigma_{i, j}^{d}-\mathbb{E}\left(\Sigma_{i}^{d}\right)\right)^{2} \text { with } \mathbb{E}\left(\Sigma_{i}^{d}\right)=\frac{1}{n_{d, i}^{\mathrm{ob}}} \sum_{j=1}^{n_{d, i}^{\mathrm{ob}}} \sum_{i, j}^{d}
$$




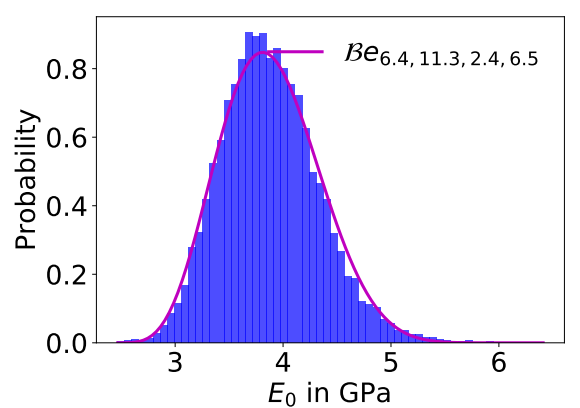

Figure 7: Distribution of $E_{0}$ obtained by MCMC sampling and its approximated beta distribution.

\subsubsection{Prior distribution}

The prior distribution of $\boldsymbol{\vartheta}=\left[E_{0}, \sigma_{\mathrm{Y} 0}, h, m_{1}, m_{2}, A r\right]$ is constructed from 6 independent distributions, as

$$
\pi_{\text {prior }}(\boldsymbol{\vartheta})=\mathcal{B} e^{E_{0}} \times \mathcal{U}^{\sigma_{Y 0}} \times \mathcal{U}^{h} \times \mathcal{U}^{m_{1}} \times \mathcal{U}^{m_{2}} \times \mathcal{U}^{A r}
$$

For all the parameters, at the exception of $E_{0}$, by lack of information we use uncorrelated uniform distributions $\mathcal{U}$ whose bounds are the ones used in the NNW training and were justified in Section 3, see Table 1. For the matrix Young's modulus $E_{0}$, we consider a beta distribution $\mathcal{B} e$ since in [16] such a distribution was used to describe the material discrepancy in the linear range, resulting in a conditional beta distribution $\mathcal{B} e\left(E_{0} ; \alpha, \beta, a, b\right)$ in which $\alpha, \beta$ are its shape parameters and $a, b$ are its lower and upper bounds, respectively. Based

\footnotetext{
${ }^{1}$ For the $n_{d}^{\text {po }}$ data points extracted from one tensile sample of a defined unique loading path, the measured stresses $\Sigma_{i}^{d}$ at different strain levels $\varepsilon_{i}^{d}$ are correlated: the correlation among the experimental measurements results from the unique material properties of a given tested sample. In all generality, the likelihood function is built, using the data points from one sample, as

$$
\pi\left(\Sigma_{1}^{d}, \ldots, \Sigma_{n_{d}^{\mathrm{po}}}^{d} \mid \varepsilon_{1}^{d}, \ldots, \varepsilon_{n_{d}^{\mathrm{po}}}^{d}, \vartheta\right)
$$

Since the correlations among the measured stresses is assumed to result solely from the shared material properties, when these material properties are given, the probability distributions of the stresses become independent. As strain $\varepsilon_{i}^{d}$ has no effect on stress $\Sigma_{j}^{d}, i \neq j$, using the theory of conditional independence, the likelihood function can be factorized into $\prod_{i=1}^{n_{d}^{\mathrm{po}}} \pi\left(\Sigma_{i}^{d} \mid \varepsilon_{i}^{d}, \boldsymbol{\vartheta}\right)$.
} 
on this conditional distribution of $E_{0}$, we have

$$
\pi\left(E_{0}\right)=\int_{\alpha, \beta, a, c} \mathcal{B} e\left(E_{0} ; \alpha, \beta, a, c\right) \pi(\alpha, \beta, a, b) \mathrm{d} \alpha \mathrm{d} \beta \mathrm{d} a \mathrm{~d} b,
$$

where the distributions of $\alpha, \beta, a, b$ were inferred in [16] from experimental tests. Their integration in Eq. (35) is carried out using a MCMC sampling, and the resulting marginal distribution is eventually approximated by a beta distribution $\mathcal{B} e\left(E_{0} \mid 6.4,11.3,2.4 \mathrm{GPa}, 6.5 \mathrm{GPa}\right)$, see Fig. 7, which serves as the prior distribution of $E_{0}$.

\subsubsection{Posterior distribution}

Finally, the posterior distribution

$$
\pi_{\text {post }}\left(\boldsymbol{\vartheta} \mid \varepsilon_{\mathrm{M}}, \boldsymbol{\Sigma}\right) \propto \pi\left(\boldsymbol{\Sigma} \mid \varepsilon_{\mathrm{M}}, \boldsymbol{\vartheta}\right) \pi_{\text {prior }}(\boldsymbol{\vartheta})
$$

is evaluated using a MCMC technique, which is a random walk in the parameter space $\boldsymbol{\vartheta} \in \mathbb{R}^{7}$, as summarized in Box 2 of Fig. 6. The adaptive variant [26] of the Metropolis algorithm [27] is used, see also [16] for details.

\section{Results and Discussion}

In this section, we first ascertain the convergence of the BI before providing the posterior distribution obtained by the NNW-accelerated BI. We also show that considering the NNW as surrogate during the BI does not impact on the accuracy. Finally we show that, while the NNW-accelerated BI is manageable, using directly the two-step MFH during the BI is computationally unaffordable.

\subsection{Convergence}

The BI process described in Section 5.1 has been conducted to generate 20000 data samples from which the first 2000 samples were discarded. The convergence of the MCMC algorithm is studied in Fig. 8 in terms of the trace, i.e. the realizations in terms of the iteration number. After a few thousands of iterations, the trace has the typical fuzzy shape of a converged analysis. 


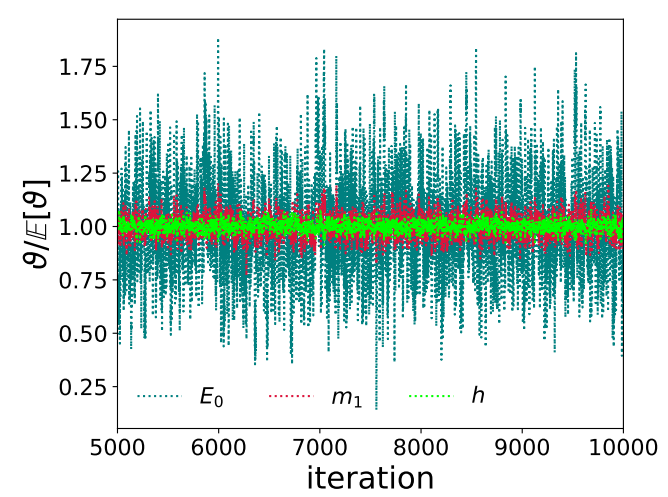

(a)

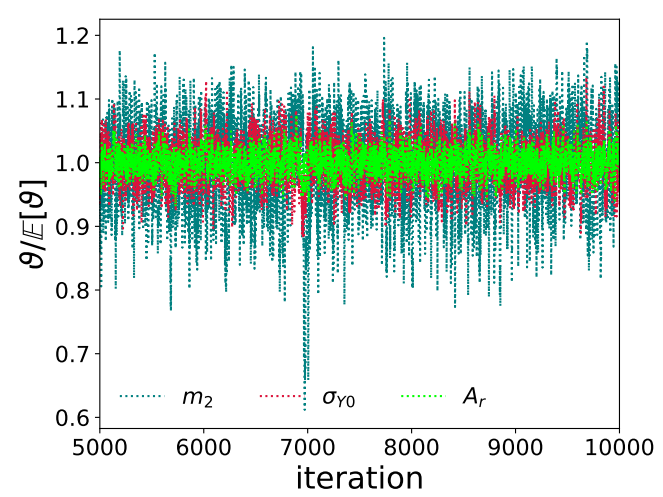

(b)

Figure 8: Trace of the inferred parameters with respect to the MCMC iteration

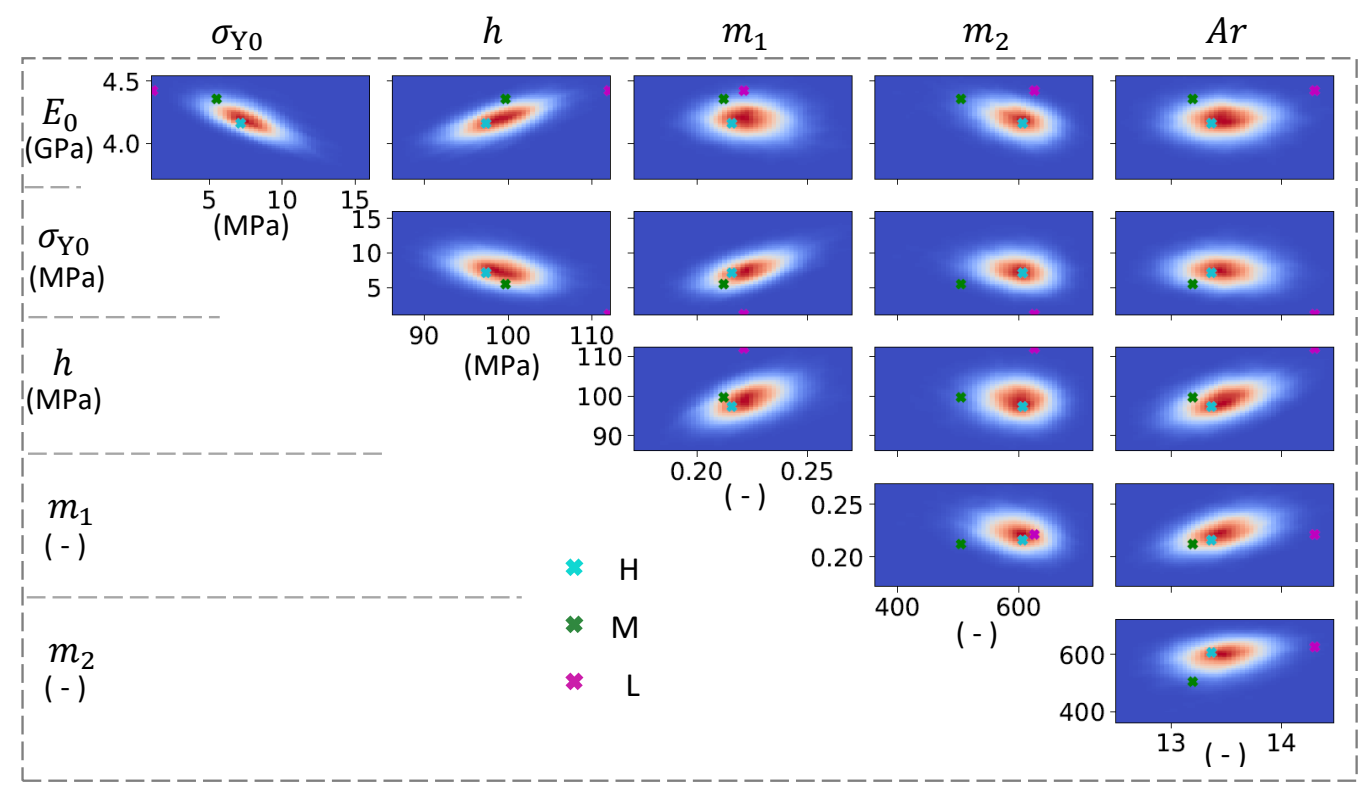

Figure 9: Distributions of material properties from the MCMC sampling; three marked points [4.16 GPa, 7.14 MPa, 97.3 MPa, 0.216, 606.7, 13.4], [4.36 GPa, 5.51 MPa, 99.7 MPa, 0.212, 504.7, 13.2], and [4.42 GPa, 1.15 MPa, 112.0 MPa, 0.221, 626.1, 14.3] are extracted and correspond to realizations of $\boldsymbol{\vartheta}$ of the Highest, Moderate and Lowest (among the 18000 samples) probabilities.

\subsection{Accuracy verification}

The posterior distribution of the random material properties $\boldsymbol{\vartheta}$ and their correlations can be seen in Fig. 9. The three data points marked in Fig. 9 were picked among the obtained random 18000 samples: the point "H", "M" or "L" represents a sample with the Highest, 


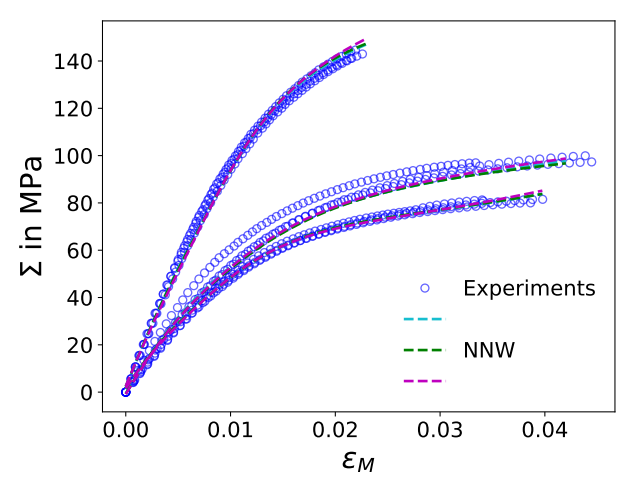

(a) NNW

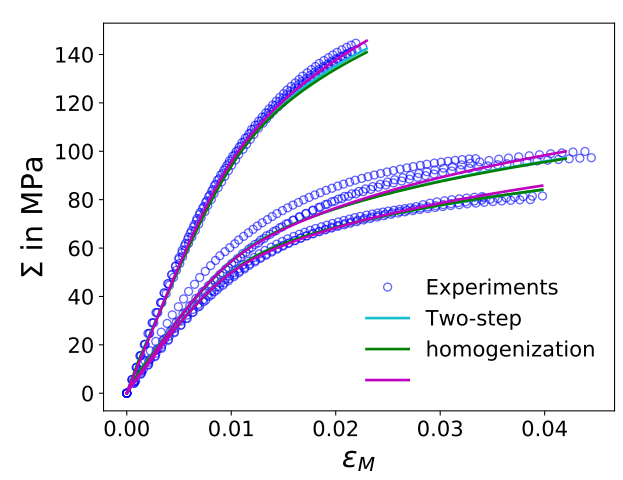

(b) Two-step homogenization

Figure 10: The comparison of the experimental measurements to the numerical prediction of (a) the NNW and (b) the two-step homogenization for the three parameters observations extracted from Fig. 9

Moderate or Lowest (among the 18000 samples) probability, respectively. For each of them, the strain-stress curves predicted by the NNW $\Phi_{\mathrm{NNM}}$ and the two-step MFH with skin-core effect $\Phi_{\mathrm{MFH}}$ are presented in Figs. 10(a) and 10(b), respectively. The colors of the curves correspond to the colors of the markers of Fig. 9. The experimental measurements are also reported for comparison purpose.

A good accuracy of surrogate $\Phi_{\mathrm{NNM}}$ is observed in Fig. 10. Among the obtained random data samples, the three samples "H", "M" and "L" exhibit a good agreement with the experimental measurements. Because of the good repeatability of the experimental measurements, the variances of the stress noise functions $\omega_{\Sigma}$, Eq. (28), are rather small. As a consequence, any sample obtained by the MCMC can serve as a good solution for the required material parameters: when using different material parameters, the discrepancy observed between the strain-stress curves is comparable to the accuracy of the trained surrogate $\Phi_{\mathrm{NNM}}$. On the one hand, rigorously speaking, the inferred distributions of material properties do not seem useful at first since the different observations provide accurate predictions. Nevertheless, on the other hand, compared to a parameters identification based on optimization techniques, instead of obtaining a unique solution, the BI reveals the non-linear relationship among the material properties, or the manifold of the material properties. Besides, it naturally allowed to considered several loading cases altogether, which could lead to three different sets of 
parameters for a deterministic inverse identification.

Nevertheless, in this paper, the micro-mechanical model is seen as deterministic: although some uncertainties exist in the identified parameters the multiscale model is not able to represent the observed dispersion in the materials. However, in the case of polymericbased composites, such dispersion can be important: in [28], PA06 tensile modulus measured at at constant temperature and strain rate ranges from 1200 to $3400 \mathrm{MPa}$, and tensile tests conducted on PA06-GF30 lead to a Young's modulus ranging from 6200 to $9500 \mathrm{MPa}$. To capture this dispersion, properties spatial distributions can be inferred [29], or BI can be adapted in order to infer the parameters of an assumed distribution of the material properties instead of the material properties themselves [30]. This so-called distribution-based BI approach requires a double MCMC sampling process in the non-linear range, which is the reason why in [16] the authors have inferred the distribution of the linear material constants only. However, because of the high efficiency, see next subsection, of the NNW, such an approach would become affordable to infer the parameters distribution of elasto-(visco)-plastic composites multiscale models.

\subsection{Computational efficiency}

Table 4 compares the computational time needed to perform a direct BI, which uses the 2-step MFH model $\Phi_{\mathrm{MFH}}$ to evaluate the likelihood, to the time to perform the NNWaccelerated BI, which uses the surrogate model $\Phi_{\mathrm{NNW}}$ when evaluating the likelihood. For the NNW-accelerated BI, an offline computation is required. Data needs first to be generated, which consists in running 500 times the 2-step MFH model $\Phi_{\mathrm{MFH}}$ along 3 loading directions. Although this requires roughly 2 days of computations, this can be perform in parallel since the simulations are independent. The NNW then needs to be trained for each loading direction, requiring half an hour. The total offline time si thus about 2 days. To perform the BI, for each accepted sample, the MCMC algorithm rejects between 10 and 20000 samples. It is estimated that 6 millions evaluations of the likelihood are required to reach the converge posterior (we note that a rejected sample requires the evaluation of the likelihood). Since evaluating the surrogate model $\Phi_{\mathrm{NNW}}$ is almost instantaneous, the NNW-accelerated BI 
Table 4: Comparison of computational time between the NNW-accelerated BI and the direct BI. Simulations were performed on a laptop.

\begin{tabular}{|c|c|c|c|c|}
\hline & \multicolumn{2}{|l|}{ Direct BI } & \multicolumn{2}{|l|}{ NNW-accelerated BI } \\
\hline Offline & & & $\begin{array}{l}\text { Number of MFH } \\
\text { simulations } \\
\text { Time of } \mathrm{MFH} \\
\text { simulations } \\
\text { Training time }\end{array}$ & $\begin{array}{c}500 \times 3 \mathrm{dir} . \\
500 \times 3 \times \\
100 \mathrm{sec}=42 \mathrm{hr} \\
8 \mathrm{~min} \times 3 \mathrm{dir} . \\
\sim 0.5 \mathrm{hr}\end{array}$ \\
\hline Online & $\begin{array}{l}\text { Number of samples } \\
\text { Number of rejected } \\
\text { iterations per sample } \\
\text { Time for one iteration } \\
\text { Estimated time for BI }\end{array}$ & $\begin{array}{c}20000 \\
{[10-20000]} \\
100 \mathrm{sec} \times 3 \text { dir. } \\
\sim 300 \times 20000 \times 3 \\
\times 100 \mathrm{sec} \simeq 57 \text { years }\end{array}$ & $\begin{array}{l}\text { Number of samples } \\
\text { Number of rejected } \\
\text { iterations per sample } \\
\text { Time for one iteration } \\
\text { Time for BI }\end{array}$ & $\begin{array}{c}20000 \\
{[10-20000]} \\
0.005 \mathrm{~s} \times 3 \text { dir. } \\
\sim 300 \times 20000 \times 3 \\
\times 0.005 \mathrm{~s} \simeq 1 \text { day }\end{array}$ \\
\hline
\end{tabular}

remains affordable and requires roughly 1 day of computation. However, the direct BI cannot be considered, since the evaluation of one likelihood requires the evaluations of the 2-step MFH model $\Phi_{\mathrm{MFH}}$ along the 3 directions, corresponding to more than 300 sec per iteration, which would lead to several years of computation. Besides, the iterations in the MCMC algorithm are dependent and the parallelization scalability is limited since the first thousands of iterations need to be burned.

\section{Conclusions}

In order to model Short Fibers Reinforced Polymer responses, a prevalent method is to adopt a multiscale modeling strategy, for which the material properties of each phase should be inferred. However, although the behavior of the composite material seen as a homogenized medium can be experimentally measured, the identification of the matrix material properties from the composites response requires a special identification process. Indeed, applying classical identification processes, such as optimization and Bayesian Inference, is limited by 
the complexity of the micromechanics models, especially for non-linear cases in which multiparameters are involved and for which the computation cost is not negligible. In particular, when considering a two-step homogenization as a non-linear multiscale model, the MCMC sampling of a BI process becomes unaffordable.

In this paper, a NNW was adopted as a surrogate model to replace the expensive micromechanics model during the material parameters identification process. The accuracy of NNW was verified from the comparison between the strain stress curves obtained with NNW and those obtained by the two-step homogenization. The matrix material properties and the effective fiber aspect ratio were then identified by the BI process from the experimental measurements on composite coupons. In particular, it was shown that the inferred properties could predict the composite material response in agreement with the experimental curves.

Although the methodology was developed for a particular homogenization method, i.e. $\mathrm{MFH}$, it can be extended to other ones, such as computational homogenization, or to more complex identification tests. For example, the method can be applied for multi-axial stressstrain cases, assuming a monotonicand proportional loading, in which case a strain vector -of size up to 6- serves as inputs, together with the material parameters, of the NNW instead of a ingle strain variable; the corresponding -up to 6-outputs will be used to represent the stress vector. Consequently, since the number of inputs is increased, the adopted structure of the NNW needs to be enhanced by more hidden layers and neurons to be able to represent a more complicated high dimensional nonlinear mapping. As a result of the enhancement of the NNW, an increased number of training data would probably be required. On the aspect of BI process, the original uni-variate Gaussian distribution used to construct the likelihood function has to be replaced by a multivariate Gaussian distribution of higher dimension -up to 6 , and the different importance of the stress entries can be addressed by using a modified precision matrix in the multivariate Gaussian distribution. Finally, in order to introduce some stochasticity in the multiscale model, it is foreseen to infer the parameters of an assumed distribution of the material properties instead of the material properties themselves. Because this so-called distribution-based BI approach requires a double MCMC 
sampling process in the non-linear range, the method is not practical with direct BI for a complex micro-mechanical model. However, because of the high efficiency of the NNW, this becomes possible in the cases of elasto-(visco)-plastic composites.

Acknowledgment. The research has been funded by the Walloon Region under the agreement no 1410246-STOMMMAC (CT-INT 2013-03-28), by the Gaitek 2015 programm of the Basque Government, and by the Austrian Research Promotion Agency (ffg) under the agreement no 850392 (STOMMMAC) in the context of the M-ERA.NET Joint Call 2014.

\section{References}

[1] K. Matous, M. G. Geers, V. G. Kouznetsova, A. Gillman, A review of predictive nonlinear theories for multiscale modeling of heterogeneous materials, Journal of Computational Physics 330 (Supplement C) (2017) $192-220$, doi:10.1016/j.jcp.2016.10.070.

[2] J. D. Eshelby, The Determination of the Elastic Field of an Ellipsoidal Inclusion, and Related Problems, Proceedings of the Royal Society of London. Series A 241 (1226) (1957) 376-396.

[3] T. Mori, K. Tanaka, Average stress in matrix and average elastic energy of materials with misfitting inclusions, Acta Metallurgica 21 (5) (1973) 571-574.

[4] R. Hill, Continuum micro-mechanics of elastoplastic polycrystals, Journal of the Mechanics and Physics of Solids 13 (2) (1965) 89 - 101, doi:10.1016/0022-5096(65)90023-2.

[5] D. R. S. Talbot, J. R. Willis, Variational Principles for Inhomogeneous Non-linear Media, IMA Journal of Applied Mathematics 35 (1) (1985) 39-54.

[6] L. Wu, L. Noels, L. Adam, I. Doghri, A combined incremental-secant mean-field homogenization scheme with per-phase residual strains for elasto-plastic composites, International Journal of Plasticity 51 (2013) 80-102, doi:10.1016/j.ijplas.2013.06.006.

[7] C. W. Camacho, C. L. Tucker, S. Yalva c, R. L. McGee, Stiffness and thermal expansion predictions for hybrid short fiber composites, Polymer Composites 11 (4) (1990) 229-239, doi:10.1002/pc.750110406.

[8] I. Doghri, L. Tinel, Micromechanics of inelastic composites with misaligned inclusions: Numerical treatment of orientation, Computer Methods in Applied Mechanics and Engineering 195 (13) (2006) 1387 - 1406, doi:10.1016/j.cma.2005.05.041.

[9] M. Vincent, T. Giroud, A. Clarke, C. Eberhardt, Description and modeling of fiber orientation in injection molding of fiber reinforced thermoplastics, Polymer 46 (17) (2005) 6719 - 6725.

[10] J. L. Beck, L. S. Katafygiotis, Updating Models and Their Uncertainties. I: Bayesian Statistical Framework, Journal of Engineering Mechanics 124 (1998) 455-461. 
[11] T. Most, Identification of the parameters of complex constitutive models: Least squares minimization vs. Bayesian updating, in: D. Straub (Ed.), Reliability and Optimization of Structural Systems, 2010.

[12] S. Madireddy, B. Sista, K. Vemaganti, A Bayesian approach to selecting hyperelastic constitutive models of soft tissue, Computer Methods in Applied Mechanics and Engineering 291 (2015) 102 - 122 , doi:10.1016/j.cma.2015.03.012.

[13] H. Rappel, L. Beex, J. Hale, L. Noels, S. Bordas, A tutorial on Bayesian inference to identify material parameters in solid mechanics, Archives of Computational Methods in Engineering doi:10.1007/s11831018-09311-x.

[14] T. C. Lai, K. Ip, Parameter estimation of orthotropic plates by Bayesian sensitivity analysis, Composite Structures 34 (1) (1996) 29 - 42, doi:10.1016/0263-8223(95)00128-X.

[15] F. Daghia, S. de Miranda, F. Ubertini, E. Viola, Estimation of elastic constants of thick laminated plates within a Bayesian framework, Composite Structures 80 (3) (2007) 461 - 473, doi: 10.1016/j.compstruct.2006.06.030.

[16] M. Mohamedou, K. Z. Uriondo, C. N. Chung, H. Rappel, L. Beex, L. Adam, Z. Major, L. Wu, L. Noels, Bayesian Identification of Mean-Field Homogenization model parameters and uncertain matrix behavior in non-aligned short fiber composites, Composite Structures Submitted.

[17] B. A. Le, J. Yvonnet, Q.-C. He, Computational homogenization of nonlinear elastic materials using neural networks, International Journal for Numerical Methods in Engineering 104 (12) (2015) 10611084, doi:10.1002/nme.4953.

[18] M. Bessa, R. Bostanabad, Z. Liu, A. Hu, D. W. Apley, C. Brinson, W. Chen, W. K. Liu, A framework for data-driven analysis of materials under uncertainty: Countering the curse of dimensionality, Computer Methods in Applied Mechanics and Engineering 320 (2017) 633 - 667, doi:10.1016/j.cma.2017.03.037.

[19] J. F. Unger, C. Könke, Coupling of scales in a multiscale simulation using neural networks, Computers \& Structures 86 (21) (2008) 1994 - 2003, ISSN 0045-7949, doi:10.1016/j.compstruc.2008.05.004.

[20] F. Fritzen, M. Fernández, F. Larsson, On-the-Fly Adaptivity for Nonlinear Twoscale Simulations Using Artificial Neural Networks and Reduced Order Modeling, Frontiers in Materials 6 (2019) 75, ISSN 22968016, doi:10.3389/fmats.2019.00075.

[21] D. R. S. Talbot, J. R. Willis, Bounds and Self-Consistent Estimates for the Overall Properties of Nonlinear Composites, IMA Journal of Applied Mathematics 39 (3) (1987) 215-240, doi: 10.1093/imamat/39.3.215

[22] P. Ponte Castañeda, A New Variational Principle and its Application to Nonlinear Heterogeneous Systems, SIAM Journal on Applied Mathematics 52 (5) (1992) 1321-1341.

[23] B. Weber, B. Kenmeugne, J. Clement, J. Robert, Improvements of multiaxial fatigue criteria computation for a strong reduction of calculation duration, Computational Materials Science 15 (4) (1999) 
381 - 399, doi:10.1016/S0927-0256(98)00129-3.

[24] F. Pedregosa, G. Varoquaux, A. Gramfort, V. Michel, B. Thirion, O. Grisel, M. Blondel, P. Prettenhofer, R. Weiss, V. Dubourg, J. Vanderplas, A. Passos, D. Cournapeau, M. Brucher, M. Perrot, E. Duchesnay, Scikit-learn: Machine Learning in Python, Journal of Machine Learning Research 12 (2011) 2825-2830.

[25] C. M. Bishop, in: Pattern Recognition and Machine Learning, Information Science and Statistics, Springer-Verlag, New York, USA, ISBN 978-0-387-31073-2, 2006.

[26] H. Haario, E. Saksman, J. Tamminen, Adaptive proposal distribution for random walk Metropolis algorithm, Computational Statistics 14 (3) (1999) 375-395, doi:10.1007/s001800050022.

[27] W. Gilks, S. Richardson, D. Spiegelhalter, Markov Chain Monte Carlo in Practice, in: Chapman, Hall (Eds.), Chapman \& Hall/CRC Interdisciplinary Statistic, Weinheim, Germany, 1995.

[28] H. Nouri, Modélisation et identification de lois de comportement avec endommagement en fatigue polycyclique de matriaux composite à matrice thermoplastique, Ph.D. thesis, Arts et Métiers ParisTech, Metz (France), 2009.

[29] A. Vigliotti, G. Csányi, V. Deshpande, Bayesian inference of the spatial distributions of material properties, Journal of the Mechanics and Physics of Solids 118 (2018) 74 - 97, doi: https://doi.org/10.1016/j.jmps.2018.05.007.

[30] H. Rappel, L. Beex, Estimating fibres material parameter distributions from limited data with the help of Bayesian inference, European Journal of Mechanics - A/Solids 75 (2019) 169 - 196. 